\title{
The true Blazhko behaviour of DM Cyg
}

\author{
Zs. Hurta ${ }^{1,2}$ \\ ${ }^{1}$ Eötvös Loránd University, Department of Astronomy, Budapest, Hungary \\ ${ }^{2}$ Konkoly Observatory, Budapest, Hungary
}

\begin{abstract}
We present preliminary results of our work on DM Cyg, an RRab star with steadily increasing pulsation period. The Blazhko modulation of the light curve of DM Cyg has not been undoubtedly confirmed yet. A reanalysis of the original data (Sódor \& Jurcsik 2005) could not confirm the $26 \mathrm{~d}$ periodicity found by Lysova \& Firmanyuk (1980) in the timings of maximum brightness data of visual observations. Neither the scarce photoelectric observations (Fitch 1966, Sturch 1966, Hipparcos 1997) nor the CCD data of the NSVS (Woźniak 2004) survey suggested a notable light curve modulation.

In order to get a definite answer whether the light curve of DM Cyg is stable or it shows any kind of modulation it was observed in the course of the Konkoly Blazhko Survey in the 2007 and 2008 seasons. Using the automated $60 \mathrm{~cm}$ telescope of the Konkoly Observatory, Svábhegy, Budapest, equipped with a Wright $750 \times 1100 \mathrm{CCD}$ camera and $B V I_{C}$ filters we obtained more than 3000 data points on about 80 nights in each band. Archive photoelectric and photographic observations obtained with the $60 \mathrm{~cm}$ telescope and a $16 \mathrm{~cm}$ astrograph of the Konkoly Observatory in 1978 and between 1934 and 1958 were also analyzed. The photoelectric and photographic photometry provided $75 \mathrm{~B}, \mathrm{~V}$ and $1031 \mathrm{pg}$ data points from 4 and 40 nights, respectively.

The CCD observations revealed that the light curve of DM Cyg is in fact modulated, but with very small amplitude. The maximum brightness variation hardly exceeds 0.05 mag in the $\checkmark$ band, while no definite phase modulation of the light curve and/or maximum timings is evident. The amplitudes of the modulation frequencies that form equidistant triplets around the pulsation frequency and its harmonics are below $15 \mathrm{mmag}$. There is some indication of light curve modulation in the Konkoly photographic data as well.

Our data confirm that DM Cyg shows Blazhko modulation but with significantly different period and character (amplitude/phase modulation) than it was found by Lysova \& Firmanyuk (2000). A detailed analysis of our observations of DM Cyg with its true Blazhko period will be submitted to MNRAS in early 2009.
\end{abstract}

Individual Objects: DM Cyg

Acknowledgments. The financial support of OTKA grants K-68626 and T-048961 is acknowledged.

\section{References}

Fitch, W. S., Wisniewski, W. Z., \& Johnson, H. L. 1966, Comm. Lunar and Planetary lab., 5, 71

Lysova, L., \& Firmanyuk, V. 1980, Ast. Cir., 1122, 3

Sódor Á., \& Jurcsik, J. 2005, IBVS, 5641 

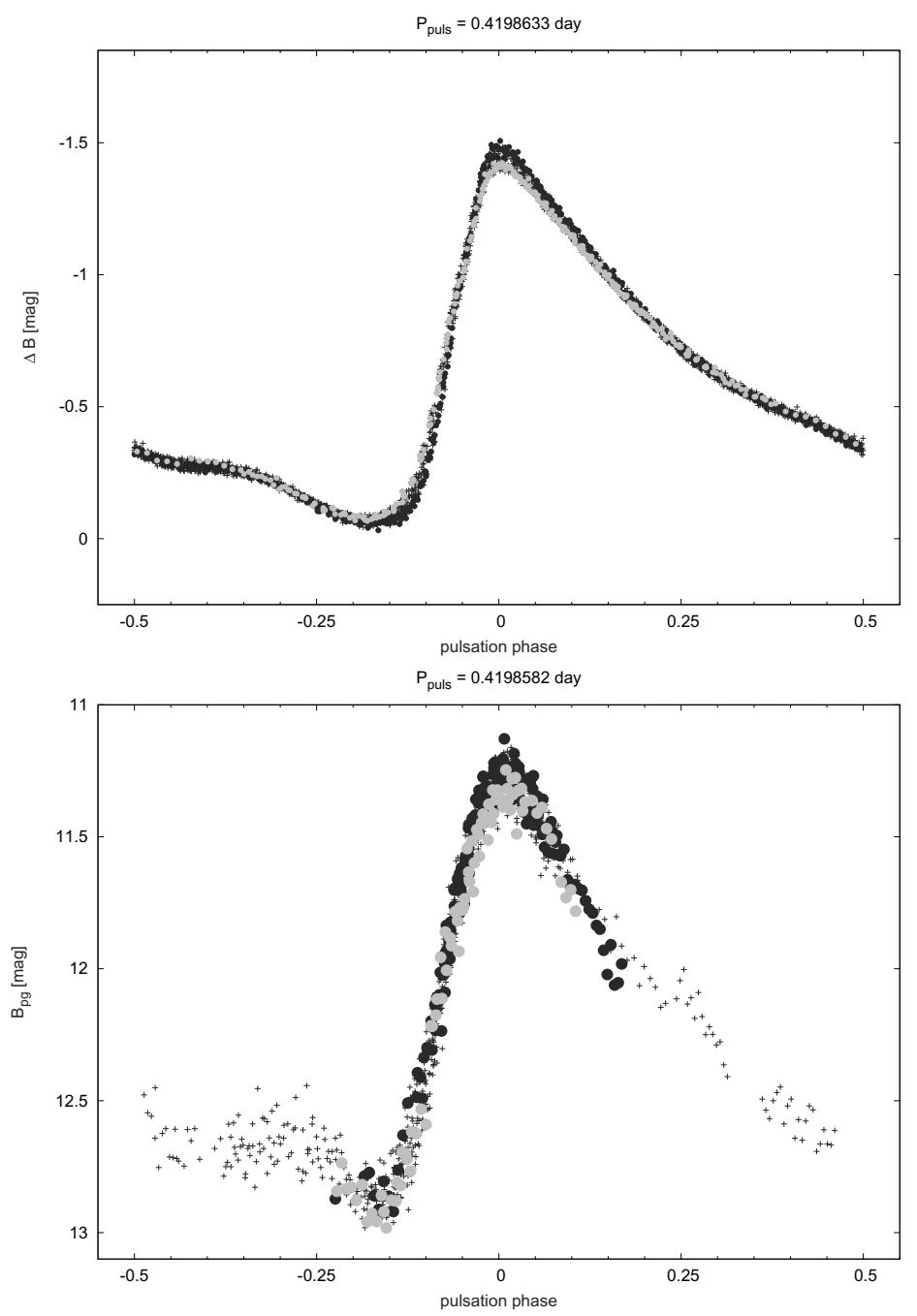

Figure 1: CCD B and the photographic light curves of DM Cyg observed with the instruments of the Konkoly Observatory. Data belonging to the largest and smallest amplitude phases of the modulation are plotted with black and grey dots, respectively. The CCD data show without doubt that the light curve of DM Cyg is not stable; the full amplitude of the pulsation in B band varies by about $0.1 \mathrm{mag}$. There is some indication that the modulation of the light curve is also present in the photographic data, but taking into account the higher noise of the photographic data and also the small amplitude of the modulation, the photographic data alone are not suitable to determine the Blazhko behaviour of DM Cyg.

Sturch, C. 1966, ApJ, 143, 774

Woźniak, P. R., Vestrand, W. T., Akerlof, W. C., et al. 2004, AJ, 127, 2436 